\title{
PERFIL DOS PACIENTES VÍTIMAS DE TRAUMA TORÁCICO ATENDIDOS NO HOSPITAL UNIVERSITÁRIO EVANGÉLICO DE CURITIBA (HUEC).
}

\author{
THORACIC TRAUMA PROFILE OF ATTENDED VICTIMS AT HOSPITAL \\ UNIVERSITÁRIO EVANGÉLICO DE CURITIBA (HUEC)
}

DOI: $10.5380 /$ rmu.v1i2.40701

Carlos Roberto Naufel Junior ${ }^{\text {; }}$ Carolina Talini ${ }^{2}$; Lydio Barbier Neto ${ }^{3}$

\section{RESUMO}

Introdução: O trauma torácico cursa frequentemente com alterações na dinâmica respiratória e circulatória. A maioria dos traumas torácicos é tratada com drenagem pleural e somente 15-30\% necessitarão de toracotomia. Objetivo: Analisar o perfil dos pacientes vítimas de trauma torácico. Metodologia: Analisados 98 prontuários entre Janeiro e Dezembro de 2011, com acometimento torácico que necessitou de drenagem pleural para seu tratamento. Resultados: Oitenta e dois pacientes eram homens e 16 mulheres. A idade média de 36,9 \pm 16 anos (variando entre 9 e 83 anos). Quarenta e três (43,8\%) foram vítimas de trauma penetrante e 55 (56,2\%) de trauma fechado. Quinze pacientes apresentaram hemotórax, 23 pneumotórax e 55 hemopneumotórax. Sessenta e oito pacientes $(69,4 \%)$ sofreram lesões associadas ao trauma torácico. A permanência com dreno foi de $7 \pm 6,2$ dias. 0 volume drenado imediatamente foi $401 \mathrm{~mL}$ (variando entre 50 e $1050 \mathrm{ml}$ ). Em 12 casos foi realizada toracotomia. Setenta (71,4\%) pacientes foram submetidos à antibioticoterapia, por $4,6 \pm 6,9$ dias. Vinte e sete receberam cuidados em unidade de terapia intensiva, por $10,3 \pm 7,9$ dias. 0 tempo médio de internamento foi $9,3 \pm 8,4$ dias (variando entre 1 e 48 dias). Trinta e um (31,6\%) pacientes apresentaram complicações e $17(17,3 \%)$ foram a óbito. Conclusão: Homens com idade entre 15 e 29 anos foram os mais acometidos, submetidos à drenagem pleural por trauma fechado, mais frequentemente associado a hemopneumotórax. A baixa incidência de toracotomia neste estudo confirma a drenagem como tratamento preferencial dos traumas torácicos em nosso meio.

Palavras-Chave: Traumatismos torácicos/epidemiologia; Traumatismos abdominais; Drenagem; Hemotórax.

\section{ABSTRACT}

Introduction: Thoracic trauma demands special attention because of the changes in respiratory and cardiac dynamics. Most thoracic traumas are treated with chest tubes, only 15-30\% require thoracotomy. Objective: To analyze the victims' profiles of thoracic trauma. Materials and methods: Analysis of 98 medical records treated in a trauma service from January to December 2011, with thoracic involvement that required pleural drainage. Results: Eighty two patients were male and 16 were female. The mean age was $36,9 \pm 16$ years (from 9 to 83 years). Forty three $(43,8 \%)$ were victims of penetrating trauma e $55(56,2 \%)$ of blunt trauma. Fifteen patients had hemothorax, 23 pneumothorax and 55 hemopneumothorax. Sixty eight patients $(69,4 \%)$ suffered associated lesions. The average length with the drain was $7 \pm 6,2$ days. The mean volume drained immediately was $401 \mathrm{~mL}$ (from 50 to $1050 \mathrm{~mL}$ ). In 12 cases thoracotomy was performed. Seventy patients $(71,4 \%)$ used antibiotic for an average of $4,6 \pm 6,9$ days. Twenty seven patients remained in intensive care unit, for an average of $10,3 \pm 7,9$ days. The mean time of hospitalization was $9,3 \pm 8,4$ days (from 1 to 48 days). Thirty one patients $(31,6 \%)$ presented complications and $17(17,3 \%)$ died. Conclusions: Thoracic trauma was more incident in men with age between 15 e 29 years, most frequently blunt trauma victims with hemopneumothorax. The incidence of thoracotomy is this study confirms pleural drainage as the first choice treatment for most of patients in our experience.

Keywords: Thoracic injuries/epidemiology; Woundings, penetrating; Abdominal injuries; Drainage; Hemothorax,

\footnotetext{
1 - Preceptor da residência de Cirurgia Geral do HUEC; cirurgião do aparelho digestivo; professor adjunto de Clínica cirúrgica I da Faculdade Evangélica do Paraná (FEPAR)

2 - Médica residente em Cirurgia Geral no HU de Cascavel-PR; médica formada pela Faculdade Evangélica do Paraná (FEPAR).

3 - Acadêmico do 11 período de medicina da Faculdade Evangélica do Paraná (FEPAR).
}

Contato do Autor / Mail to:

Lydio Barbier Neto - lydiobarbier@hotmail.com

Rua Padre Anchieta, 2770, Bigorrilho, Curitiba, Paraná CEP:807300-000 


\section{INTRODUÇÃO}

O trauma torácico é uma das principais lesões nos pacientes atendidos nos prontos-socorros, presente em até $30 \%$ dos casos e representando cerca de $20 \%$ das mortes decorrentes de traumatismos ${ }^{1}$.

Esta alta mortalidade se deve a associação do aumento da incidência desses traumas e ao poder energético e mecanismos lesivos desta lesão (maior velocidade dos automóveis e o aumento da violência interpessoal). Os traumas torácicos podem se apresentar apenas com lesões superficiais ou causar risco imediato à vida, como por exemplo: obstrução das vias aéreas, pneumotórax hipertensivo, pneumotórax aberto, hemotórax maciço, tórax instável, tamponamento cardíaco, contusão pulmonar, contusão miocárdica, ruptura aórtica, ruptura traumática do diafragma, laceração traqueobrônquica e esofágica².

Segundo Whestpal (2009), a maioria dos traumatismos torácicos é tratada prontamente com a drenagem pleural e em menor frequência evoluem para toracotomia. A drenagem torácica tem como objetivo, quando já estabelecido diagnóstico, restabelecer a pressão negativa do espaço pleural normalizando as pressões intratorácicas e facilitando o desaparecimento das atelectasias e o aumento da saturação pulmonar. Ela é responsável pela remoção de ar, líquidos e sólidos do espaço pleural ou mediastino, que podem ser resultantes neste caso do trauma. As indicações de drenagem torácica do trauma torácico são basicamente: hemotórax, pneumotórax (principalmente o do tipo hipertensivo) e hemopneumotórax. As principais indicações de toracotomia estão relacionadas ao tamponamento cardíaco, choque hipovolêmico ou insuficiência respiratória por intenso escape aéreo pelo dreno torácico ${ }^{3}$.

O trauma torácico tem grande incidência de lesões associadas de outras estruturas e órgãos, o que faz necessária uma rápida intervenção pelos desequilíbrios metabólicos consequentes ao trauma. No trauma torácico os órgãos mais acometidos são os pulmões, o coração, os grandes vasos e as vias aéreas maiores ${ }^{3}$.

De acordo com Fontelles (2000), a mortalidade dos pacientes com apenas uma lesão isolada do tórax varia de $4 \%$ a $8 \%$, e aumenta para $10 \%$ a $25 \%$, quando outro órgão é envolvido, elevando-se para 35\% quando há comprometimento de múltiplos sistemas orgânicos.

O objetivo do presente estudo é a análise do perfil dos pacientes vítimas de trauma torácico submetidos à drenagem torácica fechada quanto ao agente causador do trauma, tipo de tórax traumático, sexo e idade. De forma secundária, objetiva também analisar o lado submetido à drenagem, tempo de permanência com a drenagem, volume drenado de imediato, toracotomia, lesões associadas, antibioticoterapia, tempo de internamento total, complicações e óbitos.

\section{METODOLOGIA}

Foi desenvolvido um estudo retrospectivo através do levantamento de prontuários de pacientes atendidos em um serviço especializado em trauma da cidade de Curitiba/PR, no período compreendido entre 01 de janeiro de 2011 a 31 de dezembro de 2011.

Foram analisados 98 prontuários eletrônicos de pacientes vítimas de trauma torácico que necessitaram de drenagem pleural como um método de tratamento neste serviço e registrados: data do atendimento, idade, sexo, tipo do trauma aberto (FAF/FAB) ou fechado (agressão, colisão, queda de nível, atropelamento, esmagamento, queda de motocicleta, entre outros), lado submetido à drenagem (direita, esquerda ou bilateral), tipo de tórax traumático (pneumotórax, hemotórax, hemopneumotórax), tempo de permanência com a drenagem, volume drenado de imediato, se houve toracotomia e qual seu achado, lesões associadas, antibioticoterapia e sua duração, tempo de internamento total e em Unidade de Terapia Intensiva (UTI) e complicações e óbitos.

\section{RESULTADOS}

A amostra total foi composta por 98 pacientes vítimas de trauma que passaram pelo procedimento de drenagem torácica fechada, dos quais $16(16,3 \%)$ eram do sexo feminino e $82(83,7 \%)$ do sexo masculino. Para avaliação da faixa etária acometida, os pacientes foram subdivididos em grupos etários. Dos pacientes atendidos, dois tinham menos de 15 anos; 38 pacientes tinham idade entre 15 e 29 anos, 33 pacientes entre 30 e 44 anos, 14 pacientes entre 45 e 59 anos e, 11 pacientes tinham mais de 60 anos. A média de idade encontrada foi de 36,9 \pm 16 anos (sendo a idade mínima 9 anos e a máxima 83 anos).

Quanto ao tipo de trauma, $43(43,8 \%)$ sofreram ferimentos abertos - sendo destes, $23(53,5 \%)$ causados por arma de fogo e 20 (46,5\%) por arma branca. Os demais pacientes, totalizando 55 (56,2\%), foram vítimas de trauma fechado, os principais mecanismos envolvidos estão demonstrados na figura 1.

O hemitórax acometido foi o direito em 38 $(38,7 \%)$ pacientes, o esquerdo em $40(40,8 \%)$ pacientes e $18(18,4 \%)$ pacientes foram submetidos à drenagem bilateral. Em dois casos o hemitórax acometido não foi especificado.

Foram encontrados pacientes que apresentaram hemotórax, pneumotórax, hemopneumotórax ou até mesmo dois tipos diferentes de tórax traumáticos simultaneamente - um em cada 
hemitórax, em proporções conforme descrito na figura 2 .

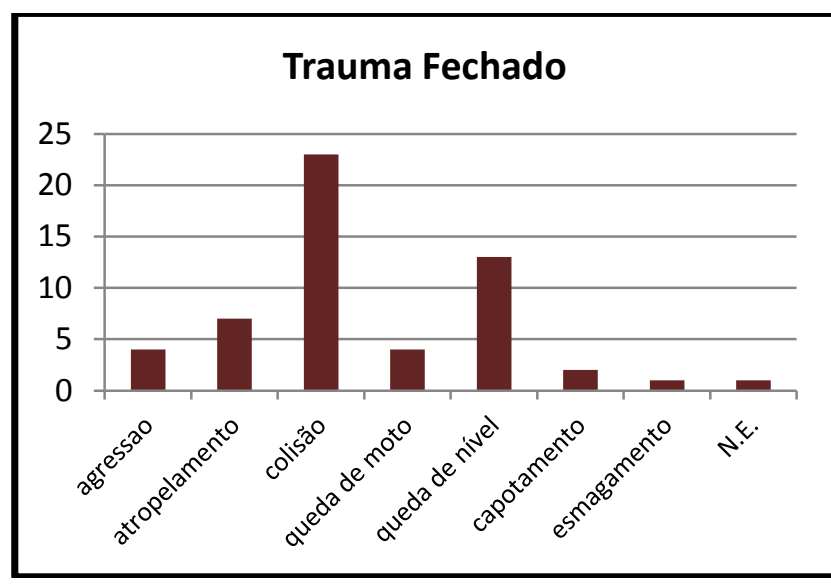

Figura 1. Mecanismos do trauma fechado

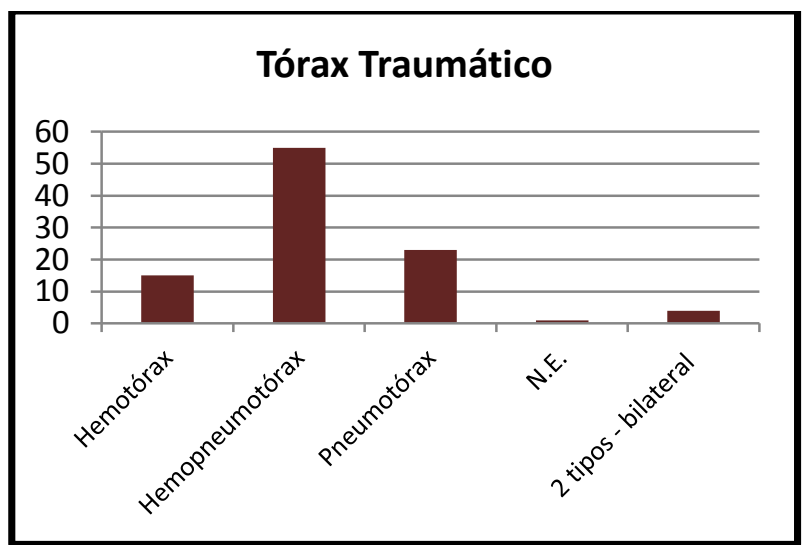

Figura 2. Distribuição do tipo de trauma torácico

O volume drenado imediatamente após instalação do dreno foi avaliado nos pacientes que apresentaram hemotórax ou hemopneumotórax, totalizando 75 pacientes. Em 30 (40\%) destes pacientes, o volume não foi especificado. Em relação aos demais, a média de volume drenado imediatamente foi de $401 \pm$ $84 \mathrm{~mL}$, sendo que o maior volume de $1050 \mathrm{ml}$ e o menor de $50 \mathrm{~mL}$.

Também foi analisado o tempo de permanência com dreno e a média encontrada foi de 7 $\pm 6,2$ dias. Um paciente permaneceu em drenagem torácica fechada (DTF) por 40 dias.

Doze $(12,25 \%)$ pacientes necessitaram de toracotomia. Em dois casos $(16,6 \%)$ a toracotomia foi feita para permitir a realização de massagem cardíaca intratorácica; lobectomia em decorrência de laceração pulmonar (dois casos); decorticação pulmonar (dois casos); toracotomia por lesões de grandes vasos - veia axilar direita e aorta torácica (dois casos). Foram também realizadas pneumorrafia devido à lesão pulmonar; toracotomia para tratamento de fístula brônquica; drenagem de empiema e janela pericárdica (um caso cada).
Quando analisadas todas as lesões ocorridas nos traumas torácicos (59 lesões - 33,15\%) houve: 29 $(22,83 \%)$ pacientes com fraturas de costela. Sessenta e oito $(69,4 \%)$ pacientes apresentaram lesões extratorácicas (119 lesões - 66,85\% das lesões ocorridas) associadas à lesão inicial que levou a necessidade de drenagem. As mais comumente encontradas foram: lesões intra-abdominais (21 $17,64 \%)$, lesões neurológicas (30 - 25,21\%) e ortopédicas (38-31,93\%).

Em relação à instituição de antibioticoterapia após a drenagem, observou-se que 70 (71,43\%) pacientes receberam algum tipo de antibiótico. 0 medicamento mais utilizado foi a cefalexina em 40 $(51,1 \%)$ pacientes e os demais seguiram a distribuição demonstrada na figura 3. O tempo médio de uso do antimicrobiano foi de 4,6 $\pm 6,9$ dias, sendo o tempo máximo de 46 dias.

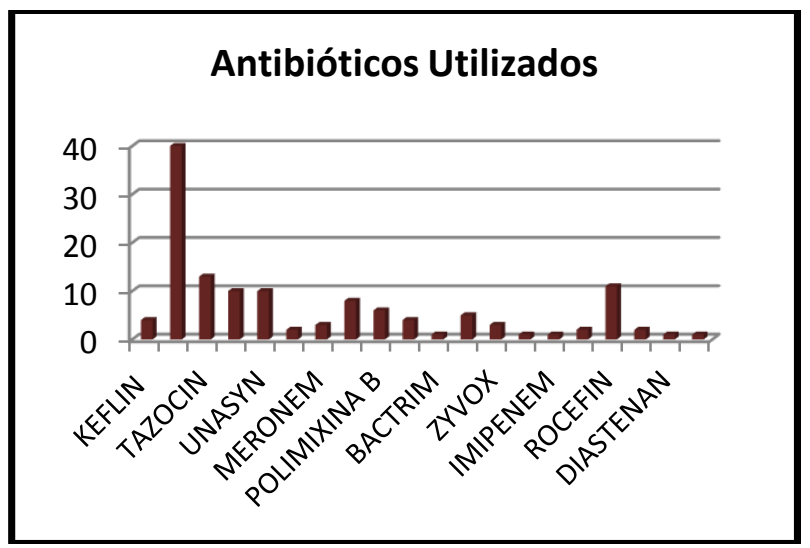

Figura 3. Antibióticos utilizados.

Vinte e sete $(27,5 \%)$ pacientes foram encaminhados para a UTI, por um tempo médio de 10,3 $\pm 7,9$ dias. $O$ tempo máximo de permanência em UTI foi de 38 dias. Em relação ao tempo total de hospitalização, observou-se uma média de $9,3 \pm 8,4$ dias, sendo o maior tempo de internamento o de 48 dias.

Entre todos os pacientes, 31 (31,6\%) apresentaram algum tipo de complicação, sendo que em 17 deles mais de uma complicação ocorreu. Foi encontrada com maior frequência a pneumonia (cinco casos - 10,4\%); embolia gordurosa (10,4\%); sepse, peritonite e choque hipovolêmico (quatro casos cada $8,3 \%$ ) além de lesões vasculares, abscessos intraabdominais e empiema (cada um com dois casos $4,2 \%)$. Dezessete $(17,35 \%)$ pacientes foram a óbito.

\section{DISCUSSÃO}

Observa-se na literatura certa concordância em relação à idade das vítimas de trauma, sendo o sexo masculino mais acometido, com uma incidência que 
varia entre $89,5 \%^{3-5}$ e $95,2 \%^{1}$. Desta forma, o presente artigo, com $83,7 \%$ dos acometidos sendo do sexo masculino, se mostra consonante com a literatura. Quanto à idade acometida observou-se maior incidência entra a população de 15 a 29 anos, em concordância com Scapolan et al. (2010), Fontelles et al. (2000) e Whestpal et al. (2009) e uma média encontrada de 36,9 anos um pouco abaixo da relatada por Cuba et al. (2005).

A maioria dos traumas ocorridos foi do tipo fechado $(56,2 \%)$ sendo a causa mais comum a colisão, fato corroborado pelos estudos de Melo et al. (2003, $80 \%$ de traumas fechados), Hunt et al. (2006,60\%), Balci et al. (2004,60\%) e Bailey et al. (2000), com 90\% de traumas fechados. Houve, entretanto, discordância em relação a outros estudos ${ }^{1,3,4,5,9}$ que apresentaram maior incidência de ferimentos penetrantes por arma branca. Quanto aos ferimentos abertos também não houve concordância com os artigos anteriormente citados, pois, neste estudo observou-se maioria de ferimentos por arma de fogo $(53,5 \%)$.

O tipo de tórax traumático mais encontrado foi o hemopneumotórax (56,12\%). Este achado é corroborado pelo estudo de Menezes et al. (2003), que demonstrou $34,2 \%$ deste tipo de tórax traumático. Outros autores encontraram predominância de hemotórax, como Melo et al. (48\%) e Fontelles et al. $(49,7 \%)$ ou pneumotórax, como na casuística de Bailey et al. (54\%).

Quando analisados os volumes drenados de imediato, encontramos concordância com outro estudo1 que apresentou uma variação de 0,0 a $1800 \mathrm{ml}$, sendo que este estudo apresentou variação entre $50 \mathrm{ml}$ e $1050 \mathrm{ml}$. O tempo médio de permanência com dreno no presente estudo foi maior do que quando comparado com outros estudos ${ }^{1,5}$, que apresentaram tempo médio de 4,8-5,6 dias e 3-7 dias respectivamente.

Neste trabalho, encontramos menor incidência de toracotomias quando comparado ao estudo de Westpahl et al. $^{3}$ - que apresentou $48,4 \%$ de toracotomias; e maior incidência do que Balci et al. ${ }^{6}$ com $8 \%$ de toracotomias. Deneuville et al. ${ }^{12}$ realizou 10 toracotomias em sua amostra, o mesmo número de toracotomias do estudo de Cuba et al. ${ }^{5}$, sendo ambas com duas a menos que neste estudo.

Diferente de alguns estudos 3,4 que revelaram predominância de trauma restrito ao tórax (61\%), o presente trabalho demonstrou maior incidência de lesões associadas $(69,4 \%)$, semelhante à casuística de Balci et al., com achado de $64,1 \%$ em sua amostra. Quanto às lesões intratorácicas, foi encontrada predominância de lesões pulmonares, assim como outros estudos ${ }^{3,11}$. Em relação à lesão torácica mais comum, encontramos $20,4 \%$ dos pacientes com fraturas em mais do que dois arcos costais, uma porcentagem muito maior que a demonstrada por Liman et al. ${ }^{13}$, com $4,7 \%$ de mais de duas fraturas de arcos costais.

Balci et al. (2004) demonstrou lesões associadas em crânio em $28,2 \%$ dos casos e Scapolan et al. (2010) revelou predominância de trauma abdominal associado ao trauma torácico. Neste estudo, a associação mais frequente foi com lesões ortopédicas. Mais de $70 \%$ dos pacientes foram tratados com antibióticos. $\mathrm{O}$ antimicrobiano mais usado neste estudo - a cefalexina - foi também o preferido por outros autores (Menezes 2003; Fontelles 2001; Cuba 2005). Em relação ao tempo de internamento, Martinus et al. (2001) encontrou 8,3 dias, tempo menor que o demonstrado aqui (10,3 dias).

A quantidade de complicações deste estudo $(31,6 \%)$ foi superior aos estudos de Westphal et al. (2009, 22,5\%), Fontelles et al. (2000, 20,9\%) e Bailey et al. (2000, 30\%). Em relação aos óbitos, este trabalho apresentou taxa de mortalidade de 17,35\%, significativamente maior do que a demonstrada por Dorgan-Neto et al. (2001, 5,8\%), Balci et al. (2004, 5,1\%), porém um pouco menor do que a demonstrada por Westphal et al., em 2009, de 20,6\%.

\section{CONCLUSÃO}

Neste estudo, homens com idade entre 15 e 29 anos apresentaram a maior prevalência de trauma torácico. O traumatismo torácico mais frequente foi o trauma fechado, o que levou a hemopneumotórax em mais da metade dos casos. O tratamento com drenagem pleural teve preferência em relação à toracotomia. $A$ maioria dos pacientes apresentou lesões extratorácicas associadas, sendo as mais comuns as ortopédicas, neurológicas e abdominais, em ordem decrescente. A taxa de mortalidade foi de $17,35 \%$.

\section{CONFLITOS DE INTERESSE}

Não houve conflito de interesses.

\section{REFERÊNCIAS}

1. FONTELLES, Mauro José Pantoja; MANTOVANI, Mario. Trauma torácico: fatores de risco de complicações pleuropulmonares pós-drenagem pleural fechada. Rev. Col. Bras. Cir., Rio de Janeiro, v. 27, n. 6, Dec. $2000 . \quad$ Disponível em:<http://www.scielo.br/scielo.php?script=sci_arttext\&pid=S0100$69912000000600008 \& \operatorname{lng}=e n \& n r m=i s o>$.

2. MELO, Alessandro Severo Alves de; MOREIRA, Luiza Beatriz Melo; MARCHIORI, Edson. Lesões traumáticas do parênquima pulmonar: aspectos na tomografia computadorizada. Radiol Bras, São Paulo, v. 36, n. 3, Jun. 2003. Disponíve em:<http://www.scielo.br/scielo.php?script=sci_arttext\&pid=S010039842003000300004\&lng=en\&nrm=iso>.

3. WESTPHAL, Fernando Luiz et al . Trauma torácico: análise de 124 pacientes submetidos à toracotomia. Rev. Col. Bras. Cir., Rio de 
Janeiro, v. 36, n. 6, Dec. 2009. Disponível em: <http://www.scielo.br/scielo.php?script=sci_arttext\&pid=S010069912009000600004\&lng=en\&nrm=iso>.

4. SCAPOLAN, Benito Maíra et al. Trauma torácico: análise de 100 casos consecutivos. Instituto de Ensino e Pesquisa Albert Einstein, São Paulo, v. 8, n. 3, Set. 2010.

5. CUBA, Ricardo Mauricio Batista Feitosa; BEZERRA, Jose Antonio Ferreira de. Traumatismo torácico: estudo retrospectivo de 168 casos. Rev. Col. Bras. Cir., Rio de Janeiro, v. 32, n. 2, Abr. 2005. Disponível em: $<$ http://www.scielo.br/scielo.php?script=sci_arttext\&pid=S0100$69912005000200002 \& \operatorname{lng}=e n \& n r m=i s o>$.

6. $\quad B A L C l$, Akin Eraslan et al. Blunt thoracic trauma in children: review of 137 cases. European Journal Cardio-Thoracic Surgery, Oxford, v. 26, n. 2 pag. 387-392. Expand+ 2004.

7. HUNT, P. A; GREAVES, I; OWENS, W. A. Emergency thoracotomy in thoracic trauma-a review. Injury, Int. J. Care Injured, v.37, n. 1 , pag. 1 - 19. 2006

8. BAILEY, R. C et al. Complications of tube thoracostomy in trauma. J. Accid. Emerg. Med., v. 17, n. 2, pag. 111-114. 2001

9. PETERSON, Richard J, et al. Pediatric and adult thoracic trauma: Age-related impact on presentation and outcome. Ann. Thoracic Surg., v. 58, n. 1, Jul. 1994

10. MENEZES, Fernanda Carrilho de et al . Sistema de drenagem torácica e uso de antimicrobianos: avaliação bacteriológica após troca do frasco coletor com seu conteúdo com 12 e 24 horas. Rev. Col. Bras. Cir., Rio de Janeiro, v. 30, n. 6, Dez. 2003. Disponível em: <http://www.scielo.br/scielo.php?script=sci_arttext\&pid=S010069912003000600005\&lng=en\&nrm=iso>.

11. DORGAN NETO, Vicente; SAAD JUNIOR, Roberto; RASSLAN, Samir. Videotoracoscopia no trauma de tórax. Rev. Col. Bras. Cir., Rio de Janeiro, v. 28, n. 1, Fev. 2001. Disponível em: $<$ http://www.scielo.br/scielo.php?script=sci_arttext\&pid=S0100$69912001000100002 \& \operatorname{lng}=e n \& n r m=i s o>$.

12. DENEUVILLE, M. Morbidity of percutaneous tube thoracostomy in trauma patients. Eur. journal. Cardiothorac. Surg., Oxford, v. 22, n. 5, pag. 673-8, Nov. 2002.

13. LIMAN, Tuba Serife et al. Chest injury due to blunt trauma. European Journal Cardio-Thoracic Surgery., Oxford, v. 23, n. 3 pag. 374-378. Expand+ 2003.

14. FONTELLES, Mauro José; MANTOVANI, Mario. Trauma torácico: importância da antibioticoterapia sobre o tempo de internação. Acta Cirurgica Brasileira, Rio de Janeiro, v. 16, n. 3, pag 133 -138 , Set. 2001.

15. MARTINUS, Richter et. al. Correlation between Crash Severity, Injury Severity, and Clinical Course in Car Occupants with Thoracic Trauma: A Technical and Medical Study. Journal of TraumaInjury Infection \& Critical Care, Denver, v. 51, n. 1, pag 10-16, Jul. 2001.

16. MANLULU, A. V.; LEE,T. W.; THUNG, K. H.; WONG, R.; YIM; A. P. C. Current indications and results of VATS in the evaluation and mangement of hemodynamically stable thoracic injuries. European Journal Cardio-Thoracic Surgery, Oxford, v. 25, n. 6, pag 1048-53. 2004. 